\title{
Robust N-Dimensional Orientation Estimation using Quadrature Filters and Tensor Whitening
}

\author{
Hans Knutsson Mats Andersson \\ Department of Electrical Engineering, Computer Vision Laboratory \\ Linköping University, S-581 83 Linköping, Sweden \\ Phone: +46 13 281887, Fax: +46 13 138526, email: knutte@isy.liu.se
}

\begin{abstract}
In this paper it is shown how estimates of local structure and orientation can be obtained using a set of spherically separable quadrature filters. The method is applicable to signals of any dimensionality the only requirement being that the filter set spans the corresponding orientation space. The estimates produced are 2:nd order tensors, the size of the tensors corresponding to the dimensionality of the input signal.

A central part of the algorithm is an operation termed 'Tensor Whitening' reminiscent of classical whitening procedures. This operation compensates exactly for any biases introduced by non-uniform filter orientation distributions and/or non-uniform filter output certainties. Examples of processing of 2D-images, 3D-volumes and 2D-image sequences are given. Sensitivity to noise and missing filter outputs are analyzed in different situations. Estimation accuracy as a function of filter orientation distributions are studied. The studies provide evidence that the algorithm is robust and preferable to other algorithms in a wide range of situations.
\end{abstract}

\section{Introduction}

Most signal analysis methods can be seen as a way of expressing the input signal in a different basis than that in which it is given. The fundamental assumption is that the original representation of a particular neighbourhood, e.g. the values of the signal for each pixel, is not a good one but that the neighbourhood can be better understood when expressed in terms of a set of carefully chosen new basis functions. The original basis is considered to be fixed, and thus the change of base be- comes trivial. However, the assumption that the data is represented in a fixed basis is often false and neglecting to note this fact can introduce severe errors.

The most prominent example of a 'changing basis' situation is the missing (or obviously erroneous) signal case. A missing signal means that one basis function is missing and not that the coordinate for that basis function is zero. In a changing basis situation proper signal analysis is possible only if the representation of the signal is complete, i.e. the representation includes not only the coordinates of the signal but also the basis in which the coordinates are given. A new method, termed normalized convolution, for handling such situation has recently been presented [6]. In this paper the basic principles of tensor whitening used in this method have been applied to the problem of robust multi-dimensional local structure and orientation estimation [5]. To be able to easily change filter orientation distributions the filters were synthezised using methodes described in [1].

The method is inherently robust and the basic principles have the potential of greatly facilitating the design of efficient fault tolerant systems. One important basic principle of the method is that certainties accompany all signals. In the present case the certainties reflect the expected reliability of individual filter outputs and missing filter outputs can be handled simply by setting the corresponding certainty to zero. Consistent with the philosophy of this paper each orientation estimate is accompanied by a certainty estimate to be used if further processing is needed. The present work should be taken as a pointer as to how adaptive robust estimation mechanisms could be implemented in general and in particular in the context of self organization and neural nets. 


\section{Theory}

The estimation algorithm can be summarized by:

$$
\mathbf{T}=\left[\sum_{k=1}^{K} c_{k} \hat{\mathbf{N}}_{k} \otimes \hat{\mathbf{N}}_{k}\right]^{+} \sum_{k=1}^{K} c_{k} q_{k} \hat{\mathbf{N}}_{k}
$$

where:

$\mathbf{T} \quad$ is a 2 :nd order tensor describing the local structure and orientation.

$K \quad$ is the number of quadrature filters.

$c_{k} \quad$ is a the certainty of quadrature filter $k$.

$\hat{\mathbf{N}}_{k} \quad$ is a normalized 2:nd order tensor corresponding to the orientation of quadrature filter $k$.

$q_{k} \quad$ is the magnitude of the output of quadrature filter $k$.

$\otimes \quad$ denotes the outer product.

[]$^{+}$denotes the Moore Penrose inverse [7].

The normalized tensors $\hat{\mathbf{N}}_{k}$ are given by:

$$
\hat{\mathbf{N}}_{k}=\hat{\mathbf{n}}_{k} \hat{\mathbf{n}}_{k}^{T}
$$

where $\hat{\mathbf{n}}_{k}$ is the filter orienting vector for quadrature filter $k$.

\subsection{Quadrature filters}

The quadrature filters are spherically separable and are defined in the Fourier domain by:

$$
F_{k}(\mathbf{u})= \begin{cases}R(\|\mathbf{u}\|)\left(\hat{\mathbf{u}} \cdot \hat{\mathbf{n}}_{k}\right)^{2} & \text { if } \mathbf{u} \cdot \hat{\mathbf{n}}_{k}>0 \\ 0 & \text { otherwise }\end{cases}
$$

where $\mathbf{u}$ is the frequency variable, $R()$ is an arbitrary non-negative function and $\hat{\mathbf{n}}_{k}$ is a normalized vector giving the orientation of quadrature filter $k$. The minimum number of filters required is given by:

$$
K \geq \frac{1}{2} d(d+1)
$$

where $d$ is the dimensionality of the input signal.

\subsubsection{Filter synthesis}

The quadrature filters used to produce the results presented in this paper were synthesized according to a
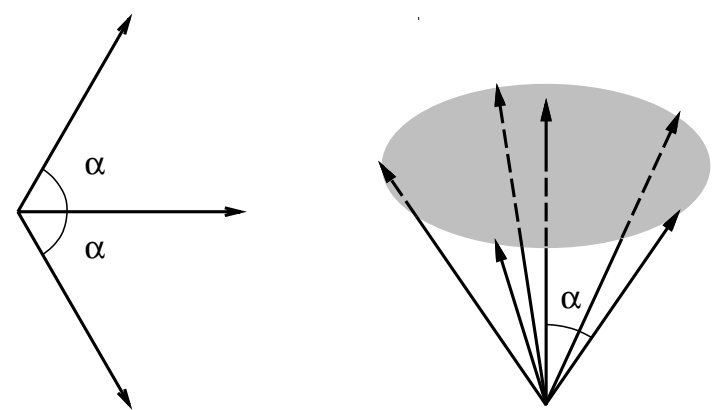

Figure 1: Figure showing the filter orientation dependence on the parameter $\alpha$ in $2 D$ (left) and $3 D$ (right).

method presented in [1]. This method allows synthetization of multi dimensional quadrature filters in any orientation using a fixed set of basis filters. The number of basis filters used to synthesize the quadrature filters was 7 for the $2 \mathrm{D}$ case and 16 for the $3 \mathrm{D}$ case.

\section{Processing examples}

To test algorithm performance a number of different situations in both 2D and 3D have been examined.

\subsection{Error measure}

The angular RMS error measures $\Delta \varphi$ were, for both the $2 \mathrm{D}$ and the $3 \mathrm{D}$ case, produced by:

$$
\Delta \varphi=\arcsin \left(\sqrt{\frac{1}{2 L} \sum_{l=1}^{L}\left\|\hat{\mathbf{x}} \hat{\mathbf{x}}^{T}-\hat{\mathbf{e}}_{1} \hat{\mathbf{e}}_{1}^{T}\right\|^{2}}\right)
$$

where:

$\hat{\mathbf{x}}$ is a unit vector in the correct orientation,

$\hat{\mathbf{e}}_{1}$ is the eigenvector corresponding to the largest eigenvalue of the estimated tensor $\mathbf{T}$,

$L$ is the number of points and

$\Delta \varphi$ is the angular RMS error.

The first example is two dimensional and uses a testpattern that consists of a circle. It has been shown that the minimum number of quadrature filters needed to estimate $2 \mathrm{D}$ orientation is three and that unbiased estimates can be obtained directly by summation if the filter orientations are $60 \mathrm{deg}$. apart [3, 5]. In figure 2 it is shown that good orientation estimation is possible 


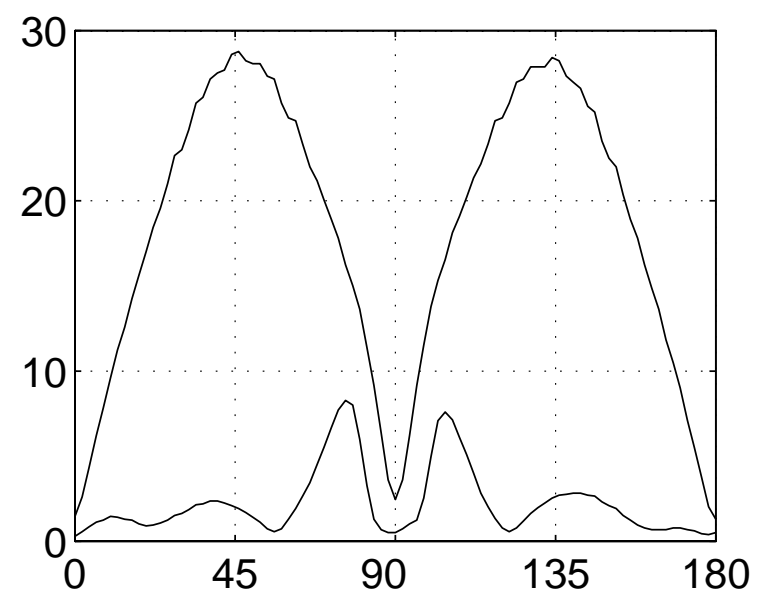

Figure 2: The angular estimation error, $\Delta \varphi$, in degrees as a function of the correct angle for a noise free signal. The filter orientations are -10,0 and $10 \mathrm{deg}$. The top curve shows the error if the tensor whitening procedure is not used, the bottom one if it is.

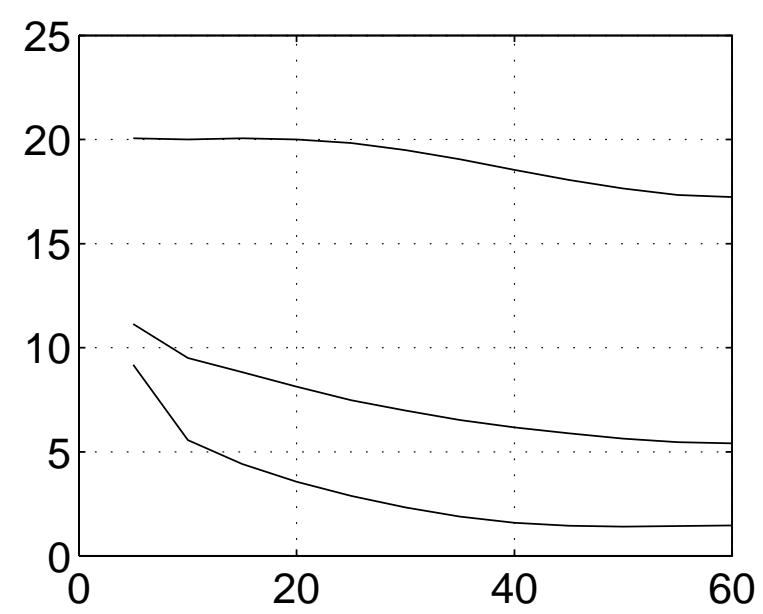

Figure 3: The 2D average angular estimation error, $\Delta \varphi$, in degrees as a function of the angle, $\alpha$, between the filters. Noise free case - bottom curve , $10 \mathrm{~dB} S N R$ - middle curve and $0 d B S N R$ - top curve.

even if the filter orientations are far from optimal if tensor whitening is used. For these results the filters were $10 \mathrm{deg}$. apart, i.e. $\alpha=10 \mathrm{deg}$, see figure 1 . The top curve shows the result for the same filter orientation distribution without tensor whitening.

In figure 3 the estimation error is shown as a function of the angle between the filters for a circular testpattern at three different SNR levels. The filters are oriented at angles 0 and $+/-\alpha$ as indicated in figure 1 (left).

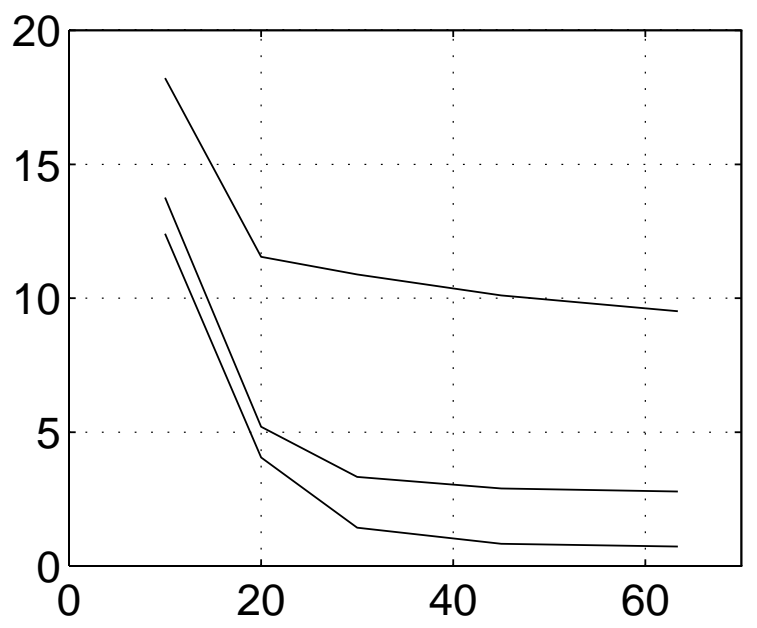

Figure 4: The 3D average estimation error, $\Delta \varphi$, in degrees for different filter distributions as a function of $\alpha$, see figure 1. Top curve - $0 d B S N R$, middle curve $10 d B$ SNR and noise free case - bottom curve

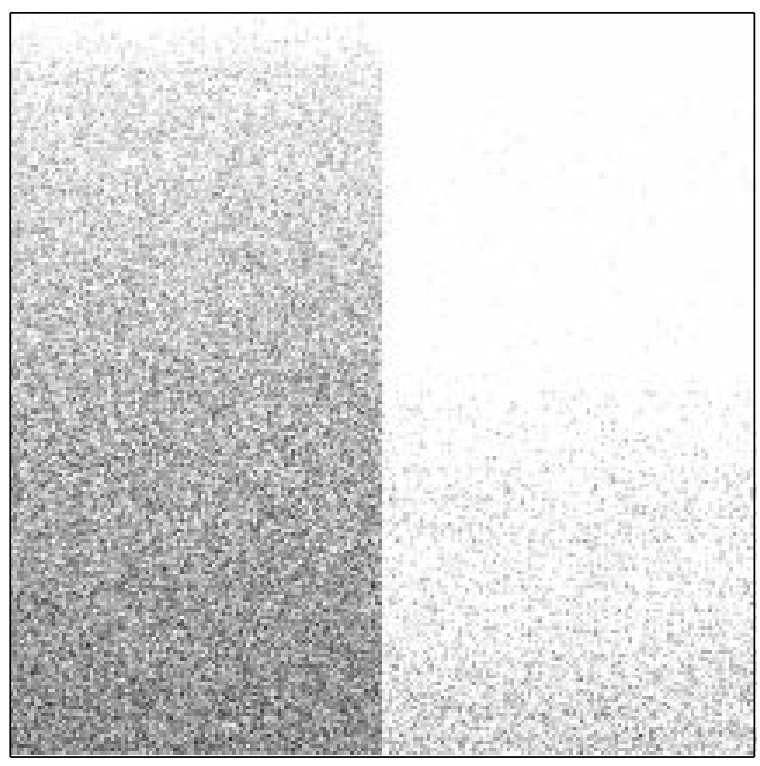

Figure 5: Figure showing the angular estimation error, $\Delta \varphi$, with (right) and without (left) tensor whitening.

Figure 4 is the equivalent of figure 3 for the three dimensional case. Results have been calculated for five different filter distributions, $\alpha=10,20,30,45$ and $63.4 \mathrm{deg}$. see figure 1 (right). The $63.4 \mathrm{deg}$. case corresponds to a uniform distribution of filters over $3 \mathrm{D}$ orientation, for a detailed analysis of this case see $[4,5,2]$. The final example should be viewed in a neural net context and is intended to demonstrate how malfunction- 


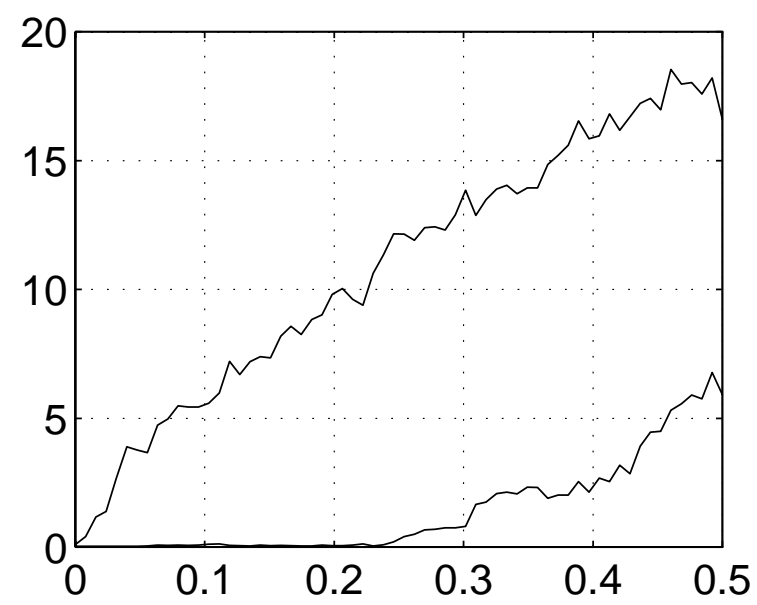

Figure 6: Figure showing the average angular estimation error, $\Delta \varphi$, as a function of filter 'drop out' probability. Bottom curve - with tensor whitening. Top curve - whithout tensor whitening.

ing units can be handled using tensor whitening. The example given is 2-dimensional and six quadrature filters uniformly distributed in orientation is used. Each filter has an equal but independent probability of malfunctioning, i.e. $c_{k}=0$ in equation 1 . At the top of figure 6 the probability is zero and increases linearly to 0.5 at the bottom of the figure. In this case it simply consisted of vertically smeared white noise. As can be seen the tensor whitening procedure is capable of handling a quite high probability of filter 'drop out'. The reason for this robustness is that, to obtain an estimate which is accurate to within the quality of the synthesized filters, it it sufficient that three filters have outputs. Consequently, using more than six filters would increase the robustness even further. Figure 5 shows the average estimation error as a function of filter drop out probability.

\section{Acknowledgment}

The support from the Swedish National Board for Technical Development is gratefully acknowledged. Part of this work has been founded by the VAP project within the Esprit Basic Research Action. The authors also wish to thank the members of our computer vision group, in particular Prof. Gösta Granlund for many inspiring discussions.

\section{References}

[1] M. Andersson. Controllable Multidimensional Filters in Low Level Computer Vision. PhD thesis, Linköping University, Sweden, S-581 83 Linköping, Sweden, September 1992. Dissertation No 282, ISBN 91-7870-981-4.

[2] M. T. Andersson and H. Knutsson. Controllable 3-D Filters for Low Level Computer Vision. In Proceedings of the 8th Scandinavian Conference on Image Analysis, Troms $\varnothing$, May 1993. SCIA.

[3] H. Knutsson. Filtering and Reconstruction in Image Processing. $\mathrm{PhD}$ thesis, Linköping University, Sweden, 1982. Diss. No. 88.

[4] H. Knutsson. Producing a continuous and distance preserving 5 -D vector representation of $3-\mathrm{D}$ orientation. In IEEE Computer Society Workshop on Computer Architecture for Pattern Analysis and Image Database Management - CAPAIDM, pages 175-182, Miami Beach, Florida, November 1985. IEEE. Report LiTH-ISY-I-0843, Linköping University, Sweden, 1986.

[5] H. Knutsson. Representing local structure using tensors. In The 6th Scandinavian Conference on Image Analysis, pages 244-251, Oulu, Finland, June 1989. Report LiTH-ISY-I-1019, Computer Vision Laboratory, Linköping University, Sweden, 1989.

[6] H. Knutsson and C-F. Westin. Normalized and Differential Convolution: Methods for Interpolation and Filtering of Incomplete and Uncertain Data. In Proceedings of IEEE Computer Society Conference on Computer Vision and Pattern Recognition, New York City, USA, June 1993. IEEE.

[7] C. R. Rao and S. K. Mitra. Generalized inverse of matrices and its applications. Wiley and Sons, New York, 1971.

[8] C-F. Westin. Feature extraction based on a tensor image description, September 1991. Thesis No. 288, ISBN 91-7870-815-X. 